\title{
Pregnancy and crash risk study wins Bruce Squires Award
}

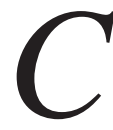

$M A J$ has awarded its top research honour to the authors of a study that showed women face an increased risk of serious car accidents during pregnancy.

Dr. Donald Redelmeier and colleagues are the recipients of the Bruce Squires Award for their article "Pregnancy and the risk of a traffic crash," which showed that pregnant drivers were $42 \%$ more likely to have a serious collision that resulted in an emergency department visit. It generated the most public interest of any $C M A J$ research paper in 2015.

The award is named for the late Dr. Bruce Squires, who was CMAJ's editorin-chief from 1989-96, and recognizes research that promotes high standards of reporting and evidence-based medicine. Sharon May of the University of Toronto, Deva Thiruchelvam of the Institute for Clinical Evaluative Sciences (ICES) and Dr. Jon Barrett of Sunnybrook Research Institute in Toronto coauthored the study.

"The study tackles an unusual question that is nevertheless highly relevant to pregnant women," says Dr. John Fletcher, CMAJ editor-in-chief. "It uses one of Canada's most valuable sources of research data, the ICES linked databases, and exemplifies careful attention to methods."

Redelmeier, the lead investigator and a senior scientist at Sunnybrook Research Institute, says he was irked by how little attention is paid to "mundane risks" during pregnancy. Patients would often ask him about exotic risks - from scuba diving to bear attacks - but seldom about everyday dangers like distracted driving.

"The best research isn't just based on introspection and inspiration, but there's also this tinge of indignation," he explains. "You're not quite satisfied with the way things are, and instead of grumbling in the back of the cafeteria, you decide to affirm or refute your constructive suggestion about how the world could be a better place."

Redelmeier's team analyzed the health records of 507262 Ontario women who gave birth between Apr. 1, 2006, and Mar. 31, 2011. The researchers found that the risk of a serious crash peaked in

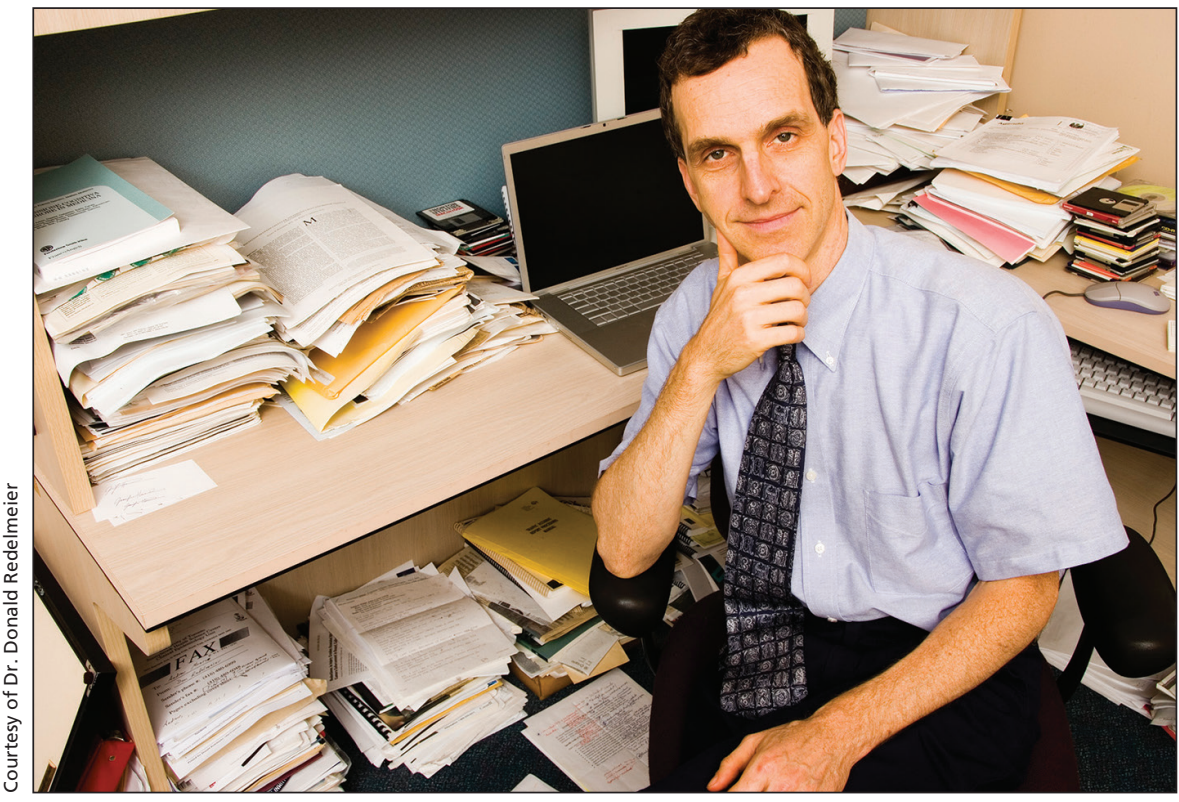

Dr. Donald Redelmeier led a study that linked pregnancy to increased risk of traffic crashes.

the fourth month of pregnancy, and was higher in the afternoon and in complicated traffic. It affected pregnant women regardless of their background, whether they had been pregnant before, or whether they were carrying a boy or a girl, among a host of other factors.

According to Redelmeier, these findings strongly suggest that pregnancy itself contributed to the risk of a crash. He argues this could be the result of "normal physiological changes" that women experience during pregnancy, like fatigue and trouble focusing.

The increased risk "could be entirely avoided by following the standard safety advice," Redelmeier adds. "Avoiding excessive speed, signalling your turns, yielding right of way, stopping at stop signs and minimizing distractions: it seems banal and yet a gentle reminder by a physician during the second trimester might do some good."

Banal or not, the advice stirred debate about the existence of "baby brain" and whether women ought to drive during pregnancy. Redelmeier says that he and coauthors were surprised by the media sensation and misunderstanding provoked by their study.

"This is solely a reason to drive more carefully," he says. "In terms of absolute risk, it's still far more dangerous to be driven by a male of the same age."
The Society of Obstetricians and Gynaecologists of Canada is now considering incorporating road safety into prenatal care guidelines. That would be a first in Canada, and possibly worldwide, says Redelmeier.

Another first: Ontario may start to track the number of pregnant women involved in traffic crashes, he adds. "That's not been part of the statistics that come out of Transport Canada, the National Highway Traffic Safety Administration or many of the statistics compiled by the World Health Organization."

Redelmeier says these early signs of change, and recognition by CMAJ, give him a "wonderful sense of validation."

"I'm really thankful to CMAJ for having the courage to publish something that was a bit unusual."

Redelmeier's team is now studying how a mother's car accident during pregnancy impacts the long-term health of her child, "particularly whether it might be one of many contributors to cerebral palsy."

"No one knows the ultimate implications for the child," he says, "but Canada is one of the few places where it's possible to research such long-term outcomes." — Lauren Vogel, CMAJ 